\title{
MMSE-Based Local ML Detection of Linearly Precoded OFDM Signals
}

\author{
L. Rugini, P.Banelli \\ Dept. of Elect. and Inform. Eng. (D.I.E.I.) \\ University of Perugia \\ Perugia, Italy \\ \{rugini, banelli\}@diei.unipg.it
}

\author{
G. B. Giannakis \\ Dept. of ECE \\ University of Minnesota \\ Minneapolis, MN \\ georgios@ece.umn.edu
}

\begin{abstract}
Linear precoding is a well known effective technique to boost the performance of orthogonal frequency-division multiplexing (OFDM) systems. A drawback of linearly precoded OFDM (LP-OFDM) systems is the high computational complexity required by maximum-likelihood (ML) detection, which is mandatory to capture all the channel diversity. Conversely, lowcomplexity techniques, such as the linear minimum meansquared error (MMSE) detection, suffer from non-negligible performance loss with respect to the ML performance. In this paper, we propose a detection technique that performs a local ML (LML) search in the neighborhood of the output provided by the MMSE detector. The trade-off between performance and complexity of the proposed LML-MMSE detector, which fall between the ones of the MMSE and ML detectors, can be nicely adjusted by appropriately setting the neighborhood size. Simulation results show that the LML-MMSE detector with minimum neighborhood size outperforms a block decision-feedback equalization (DFE) approach, while preserving a similar complexity.
\end{abstract}

Keywords-OFDM; linear precoding; MMSE; local maximumlikelihood

\section{INTRODUCTION}

Orthogonal frequency-division multiplexing (OFDM) is a widely employed technique for wireless communications over multipath Rayleigh fading channels [1]. OFDM systems generally adopt a cyclic prefix to transform the frequency-selective fading channel into a set of parallel frequency-flat channels, which facilitates the decoding and the equalization steps. However, the simplified (scalar) equalization step is coupled with a loss of multipath diversity, leading to poor bit-error rate (BER) performance. The multipath diversity is usually recovered by using forward-error correction (FEC) coding [1], at the expense of some data rate reduction.

A different approach, which can also be combined with standard FEC coding, consists of exploiting the multipath diversity by means of a linear precoder. In a linearly precoded OFDM (LP-OFDM) system, multipath diversity is introduced by transmitting different linear combinations of uncoded symbols over different subcarriers [2]. Consequently, differently from an uncoded OFDM system, the presence of a deep fade in the frequency domain does not annihilate any transmitted sym-

This work was partially supported by the Italian Minister of University and Research under the project "MC-CDMA: an air interface for the 4th generation of wireless systems." bol, but only affects the linear combination transmitted in the faded subcarrier. Therefore, by exploiting the finite-alphabet property of the constellation, the transmitted symbols can still be recovered from the data received on the other subcarriers, giving rise to improved BER performance with respect to a non-precoded system. If a non-redundant precoder is chosen [3], the performance gain is obtained without sacrificing the data rate, as firstly proposed by [4] for single-carrier flat-fading links.

In order to recover the LP-OFDM data, various detection techniques can be applied. Each of these techniques presents different complexity versus BER tradeoffs. The maximumlikelihood (ML) detector is able to get both the diversity and coding gain furnished by frequency-selective Rayleigh fading channels, thus providing good BER performance. However, its computational complexity is exponential in the precoder size. On the contrary, linear detectors and decision-directed schemes, such as the minimum mean-squared error (MMSE) detector and the decision-feedback equalization (DFE), exhibit lower complexity, but suffer from BER performance loss with respect to the ML detector.

To reduce the performance gap between linear and ML detectors, various advanced techniques could be employed, such as sphere decoding (SD) [5], semidefinite programming (SDP) [6], and probabilistic data association (PDA) [7]. These techniques approach the ML performance, with a complexity that, although much smaller than for ML, is higher than for linear and decision-directed detectors.

In this paper, we introduce the local ML (LML) detection [8]-[10] for LP-OFDM. The corresponding detector performs a complexity-constrained ML search in the neighborhood of an initial estimate. We show that the output of the MMSE detector is a convenient choice for such an initial estimate. By adjusting the neighborhood size, the proposed LML-MMSE detector can nicely trade performance for complexity, filling the gap between the MMSE and the ML detectors. Simulation results in typical wireless local area network (WLAN) channels show that the proposed LML-MMSE detectors outperform a DFE approach, while maintaining under control the increase of complexity.

\section{LP-OFDM SYSTEM MODEL}

An OFDM system with $N$ subcarriers and a cyclic prefix of length $L$ is considered. Throughout the paper, we assume 
that the multipath channel is time-invariant, with each path characterized by a Rayleigh statistic, and that the maximum delay spread does not exceed the cyclic prefix duration. We also assume time and frequency synchronization at the receiver end. As a consequence, the $l$ th received block, after the cyclic prefix removal and the fast Fourier transform (FFT), can be expressed as [11]

$$
\underline{\mathbf{y}}[l]=\underline{\mathbf{D}} \mathbf{c}[l]+\underline{\mathbf{w}}[l],
$$

where $\mathbf{y}[l]$ is a column vector of dimension $N$, $\underline{\mathbf{D}}=\sqrt{N} \operatorname{diag}\left(\mathbf{F}_{N}\left[h_{0}, h_{1}, \ldots, h_{L-1}, 0, \ldots, 0\right]^{T}\right)$ is the $N \times N$ diagonal matrix that contains the channel coefficients on the FFT grid, $h_{i}$ is the $i$ th channel tap in the time domain, $\mathbf{F}_{N}$ is the unitary $N \times N$ FFT matrix, $\underline{\mathbf{c}}[l]$ is the $N \times 1$ vector that represents the transmitted codeword, and $\underline{\mathbf{w}}[l]$ stands for the additive white Gaussian noise (AWGN) in the frequency domain. In a LP-OFDM system, the transmitted codeword $\underline{\mathbf{c}}[l]$ is obtained by linear combination of different uncoded symbols, as expressed by $\underline{\mathbf{c}}[l]=\underline{\boldsymbol{\Phi}} \mathbf{s}[l]$, where, focusing on non-redundant precoding, $\underline{\boldsymbol{\Phi}}$ is the $\bar{N} \times N$ precoder matrix, and $\underline{\mathbf{s}}[l]$ is the $N \times 1$ vector that contains the data symbols (drawn from a generic constellation of size $M$ ), which are assumed to be independent and identically distributed with unit power.

In order to reduce the system complexity, subcarrier grouping has been proposed in [3], where the subcarriers are grouped in subsets, and the precoding philosophy is applied within each subcarrier subset. It turns out that the subcarrier grouping with maximally-separated subcarriers in each subset is the optimum one in order to preserve the maximum diversity gain, which is equal to the minimum value between the precoder size and the number of channel taps. By assuming $N=B K$, where $B$ is the number of subcarrier subsets, and $K$ is the precoder size, by properly selecting the rows of the vector $\mathbf{y}[l]$, expression (1) can be split in $B$ equivalent equations

$$
\mathbf{y}_{b}[l]=\mathbf{D}_{b} \mathbf{c}_{b}[l]+\mathbf{w}_{b}[l], \quad b=1, \ldots, B
$$

where $\mathbf{y}_{b}[l]$ is the $K \times 1$ received vector relative to the $b$ th subset, $\mathbf{D}_{b}$ is the $K \times K$ diagonal matrix containing the subcarrier gains of the $b$ th subset, and $\mathbf{c}_{b}[l]=\boldsymbol{\Theta} \mathbf{s}_{b}[l]$ represents the codeword transmitted on the $b$ th subcarrier subset. The precoder $\boldsymbol{\Theta}$, which is the same for all the subsets, can be either unitary or non-unitary [3]. Since the decoding techniques considered in this paper do not depend on the subset index $b$ and on the block index $l$, in order to simplify the notation, we rewrite (2) as

$$
\mathbf{y}=\mathbf{D} \mathbf{c}+\mathbf{w}=\mathbf{D} \Theta \mathbf{s}+\mathbf{w}=\mathbf{H} \mathbf{s}+\mathbf{w},
$$

where $\mathbf{H}=\mathbf{D} \Theta$ represents the aggregate effect of the channel and of the precoder on the uncoded symbol vector $\mathbf{s}$.

\section{OVERVIEW OF DECODING TECHNIQUES}

In order to exploit all the performance advantages of linear precoding (i.e., collecting the diversity and coding gains offered by the channel), ML detection should be performed at the receiver side. In this case, due to the AWGN nature of $\mathbf{w}$, the decision rule can be formulated as

$$
\hat{\mathbf{s}}_{\mathrm{ML}}=\underset{\mathbf{s} \in S}{\arg \max }\{\Lambda(\mathbf{s})\}
$$

where

$$
\Lambda(\mathbf{s})=2 \operatorname{Re}\left(\mathbf{s}^{H} \mathbf{H}^{H} \mathbf{y}\right)-\mathbf{s}^{H} \mathbf{H}^{H} \mathbf{H} \mathbf{s}
$$

is the log-likelihood function (LLF), and $S$ is the set of all possible transmitted symbol vectors, with cardinality equal to $M^{K}$. However, due to the high computational complexity involved in the evaluation of $M^{K}$ LLFs, some suboptimum detection schemes have been suggested by the analogy between (3) and the input-output relation of equalization problems in single-carrier systems. Consequently, simpler linear detection techniques can be applied at the receiver to obtain a soft estimate of the transmitted symbol vector, as expressed by $\tilde{\mathbf{s}}=\mathbf{G} \mathbf{y}$. By employing the zero-forcing (ZF) or the MMSE criterion, the receiver matrix $\mathbf{G}$ can be expressed by $\mathbf{G}_{\mathrm{ZF}}=\mathbf{H}^{\dagger}$, and

$$
\mathbf{G}_{\mathrm{MMSE}}=\mathbf{H}^{H}\left(\mathbf{H} \mathbf{H}^{H}+\sigma_{w}^{2} \mathbf{I}_{K}\right)^{-1},
$$

respectively, where the superscript ${ }^{\dagger}$ denotes Moore-Penrose pseudoinverse, and $\sigma_{w}^{2}$ is the variance of the elements of the AWGN vector $\mathbf{w}$.

The main drawback of linear decoding techniques is that they are unable to harness the maximum possible diversity and coding gains, giving rise to significant loss in BER performance with respect to the ML approach [3]. Therefore, in order to improve the BER performance of linear detectors, while maintaining the decoding complexity at a reasonable level, we can rely on decision-directed detectors. One possibility is to cancel the previously-detected symbols by the block DFE approach of [12][13], which is designed to minimize the meansquared error (MSE) before the decision device. A different technique, alternative to the serial cancellation provided by the DFE, is the parallel interference cancellation (PIC) approach exploited in [14] for multiuser detection of direct-sequence code-division multiple-access (DS-CDMA) signals. In this case the other $K-1$ symbols, estimated using the MMSE detector, are considered as interference terms, and their effect is subtracted in a parallel fashion.

Although more powerful than linear detectors, also decision-directed techniques experience non-negligible BER loss with respect to the ML detector. As a consequence, advanced techniques such as SD [5], SDP [6], and PDA [7], have been proposed in order to approach the ML performance with a significantly reduced complexity. However, such a complexity is still high if compared with linear and decision-directed detectors. Another negative aspect of certain techniques, such as the $\mathrm{SD}$, is that the worst-case complexity for a particular received block can be much higher than the average complexity [15]. In order to overcome these drawbacks, we consider the LML technique, which is characterized by a fixed complexity, and enables performance-complexity tradeoffs between the MMSE and the ML detectors.

\section{LOCAL ML DETECTION FOR LP-OFDM SYSTEMS}

\section{A. LML Basics}

The key idea of LML detection is that we can perform the ML search by exploring only a subset of $S$, thus reducing the computational complexity with respect to the full-search detector. Indeed, if a first estimate $\hat{\mathbf{s}}$ of the transmitted symbol vector $\mathbf{s}$ is available at the receiver, and if such an estimate is 
fairly accurate, we have a high probability to refine our estimate by restricting the ML search only to those vectors that are close (in some sense) to $\hat{\mathbf{s}}$. Such an idea is not new in telecommunication systems, and has already been exploited for multiuser detection in DS-CDMA (see, e.g., [8]-[10]). However, as it will be clarified later, the considered LP-OFDM context leads to design choices that are different from those of DSCDMA systems.

Given a symbol vector $\hat{\mathbf{s}}$ and an integer $P$ selected from $\{0, \ldots, K\}$, we define the neighborhood of $\hat{\mathbf{s}}$ of size $P$ as the set

$$
S_{P}(\hat{\mathbf{s}})=\left\{\mathbf{s} \in S \mid d_{H}(\mathbf{s}, \hat{\mathbf{s}}) \leq P\right\},
$$

where $d_{H}(\mathbf{s}, \hat{\mathbf{s}})$ denotes the Hamming distance between $\mathbf{s}$ and $\hat{\mathbf{s}}$, i.e., the number of entries of $\mathbf{s}$ that are different from the entries of $\hat{\mathbf{s}}$. We define the LML detector of size $P$ associated with $\hat{\mathbf{s}}$ as

$$
\hat{\mathbf{s}}_{\mathrm{LML}}(P)=\underset{\mathbf{s} \in S_{P}(\hat{\mathbf{s}})}{\arg \max }\{\Lambda(\mathbf{s})\},
$$

that is, the ML detector constrained to the restricted set $S_{P}(\hat{\mathbf{s}})$. In other words, the LML detector evaluates all the LLFs associated with the vectors that differ at most $P$ entries from the first estimate $\hat{\mathbf{s}}$, selecting the symbol vector $\hat{\mathbf{s}}_{\mathrm{LML}}$ that produces the highest likelihood among them.

Although in (7) other distance measures could be employed, e.g., the Euclidean distance, the Hamming distance allows us to predict exactly the cardinality of $S_{P}(\hat{\mathbf{s}})$, given by

$$
C_{P}=\sum_{i=0}^{P}(M-1)^{i}\left(\begin{array}{c}
K \\
i
\end{array}\right),
$$

which turns out to be independent of $\hat{\mathbf{s}}$. Therefore, the number of LLFs to be evaluated in (8) can be easily controlled by a convenient choice of the neighborhood size $P$. Equation (9) suggests that the LML computational complexity is polynomial in $(M-1) K$, of order equal to the neighborhood size $P$, as it will be detailed in Subsection IV-C.

One of the most interesting properties of the LML detectors is the following. that

Property 1: For any fixed initial estimate $\hat{\mathbf{s}}$, it holds true

$$
\operatorname{Pr}\left\{\hat{\mathbf{s}}_{\mathrm{LML}}(P) \neq \mathbf{s}\right\} \leq \operatorname{Pr}\left\{\hat{\mathbf{s}}_{\mathrm{LML}}(i) \neq \mathbf{s}\right\}, \quad \forall i<P,
$$

that is, the block-error probability for the LML detector of size $P$ is not higher than the block-error probabilities of all the LML detectors of smaller size.

Proof: Since $S_{P}(\hat{\mathbf{s}}) \supset S_{P-1}(\hat{\mathbf{s}})$, it holds true that $\Lambda\left(\hat{\mathbf{s}}_{\mathrm{LML}}(P)\right) \geq \Lambda\left(\hat{\mathbf{s}}_{\mathrm{LML}}(P-1)\right)$, and hence

$$
\operatorname{Pr}\left\{\hat{\mathbf{s}}_{\mathrm{LML}}(P)=\mathbf{s}\right\} \geq \operatorname{Pr}\left\{\hat{\mathbf{s}}_{\mathrm{LML}}(P-1)=\mathbf{s}\right\},
$$

which easily leads to (10).

In particular, for $i=0$, Property 1 states that applying an LML search to the output $\hat{\mathbf{s}}$ of any suboptimal detector does not produce a block-error probability increase. Therefore, in most cases, also the BER will be reduced, thus motivating the LML approach.

It should be pointed out that using the output of an LML detector as the initial estimate for another LML is quite common to many LML approaches for DS-CDMA [8]-[10]. Indeed, in DS-CDMA the number of users, which plays the role of the precoder size $K$ in LP-OFDM, can be very high, and therefore the neighborhood size is forced to a value $P=1$ to limit the complexity. As a consequence, instead of increasing $P$, LML detectors for DS-CDMA try to improve the BER performance by iterating the LML detection with $P=1$. On the contrary, in OFDM for WLAN applications, the precoder size may be very small, because the maximum diversity gain can be achieved with a precoder of size $K=L(L=16$ in WLAN scenarios [16]). Therefore, if the constellation size is not very high, LML detectors with $P=2$ are not very complex.

\section{B. LML-MMSE Detector}

In the maximizations of non-convex functions, the initialization point is often a crucial step, because an unlucky starting point can lead to a local maximum that is located very far from the global one. As a consequence, for an LML technique of size $P$, we would like an initial estimate with at most $P$ errors. However, such a condition is quite hard to guarantee even when using the ML detector. Therefore, as an alternative criterion, we could ask for a detector whose soft output vector contains at least $K-P$ entries that are close to the transmitted ones. This way we can force the LML detector to confine its search to those vectors that differ from the estimated one only on the remaining $P$ entries. If we select the MSE as the measure of closeness, and we restrict the choice among the linear detectors for complexity reasons, the detector we are looking for is, of course, the MMSE decoder. Indeed, the MMSE detector minimizes the MSE of each symbol in the data vector $\mathbf{s}$, independently of the others.

Instead of the MMSE detector, [8] adopts the ZF detector as a first stage. However, in LP-OFDM systems, this detector performs poorly in the presence of deep fades. Alternatively, [10] suggests to use a DFE approach. Although the BER for DFE is typically smaller than for MMSE detection, the DFE suffers from error propagation, a phenomenon that tends to concentrate the errors in few blocks. For this reason, the LML techniques seem to be less effective when decision-directed approaches are used as starting schemes. This fact will be confirmed by simulations.

\section{Computational Complexity Reduction}

For a constellation size $M>2$, the number $C_{P}$ of LLFs to be evaluated may be too high even when moderate values of the neighborhood size $P$ and of the precoder size $K$ are employed. A possibility is to exclude from the search those vectors whose entries are not adjacent to those of $\hat{\mathbf{s}}$. We define the constellation neighborhood of $\hat{\mathbf{s}}$ of size $P$ and distance $D$ as

$$
S_{P, D}(\hat{\mathbf{s}})=\left\{\mathbf{s} \in S_{P}(\hat{\mathbf{s}}) \mid d_{E}\left(s_{i}, \hat{s}_{i}\right) \leq D \quad \forall i=1, \ldots, K\right\},
$$

where $d_{E}\left(s_{i}, \hat{s}_{i}\right)$ represents the Euclidean distance between the $i$ th entry $s_{i}$ of $\mathbf{s}$ and the $i$ th entry $\hat{s}_{i}$ of $\hat{\mathbf{s}}$. We can then define the reduced constellation (RC) LML detector of size $P$ and distance $D$ associated with $\hat{\mathbf{s}}$ as

$$
\hat{\mathbf{s}}_{\mathrm{RC}-\mathrm{LML}}(P)=\underset{\mathbf{s} \in S_{P, D}(\hat{\mathbf{s}})}{\arg \max }\{\Lambda(\mathbf{s})\} .
$$

As an example, for quaternary phase-shift keying (QPSK), the choice $D=\sqrt{2}$ excludes from the constellation neighborhood all the vectors with $s_{i}=-\hat{s}_{i}$, thus reducing the effective size of 
the constellation from $M=4$ to $m=3$. As far as performance is concerned, assuming the MMSE as first stage, the reduced constellation approach excludes those vectors with components characterized by the highest MSEs. Such symbols should be less likely to be correct, and therefore the RC-LML-MMSE detector should present a small performance loss with respect to the LML-MMSE detector.

A second possibility to reduce the complexity is to exploit the particular structure of some precoders designed for LPOFDM. In this case, rather than reducing the number of vectors in the neighborhood set, we simplify the computation of the LLF. For instance, we may assume that the precoder matrix $\boldsymbol{\Theta}$ is chosen to be unitary, and that all its entries $[\boldsymbol{\Theta}]_{i, j}$ have modulus equal to $1 / \sqrt{K}$. This class of precoders includes those designed for linear MMSE detection [17], and those designed for ML detection [3][4], which are expressed by

$$
\boldsymbol{\Theta}=\mathbf{F}_{K} \operatorname{diag}\left(1, \alpha, \ldots, \alpha^{K-1}\right),
$$

where $K$ is a power of two and $\alpha$ satisfies the equation $\alpha^{K}=\sqrt{-1}$. In this case, the computational complexity of the LML detector with $P=1$ can be significantly reduced. This fact is explained in the following for BPSK. Letting $\hat{\mathbf{s}}+\mathbf{e}_{i}$ denote the vector obtained by flipping the $i$ th entry of $\hat{\mathbf{s}}$, where $\mathbf{e}_{i}=\left[0, \ldots, 0,-2 \hat{s}_{i}, 0, \ldots, 0\right]^{T}$, it holds true that

$$
\Lambda\left(\hat{\mathbf{s}}+\mathbf{e}_{i}\right)=\Lambda(\hat{\mathbf{s}})+2 \operatorname{Re}\left(\mathbf{e}_{i}^{T} \mathbf{H}^{H} \mathbf{y}-\mathbf{e}_{i}^{T} \mathbf{H}^{H} \mathbf{H} \hat{\mathbf{s}}\right)-\mathbf{e}_{i}^{T} \mathbf{H}^{H} \mathbf{H e} e_{i} .
$$

Since $\mathbf{e}_{i}$ is non-zero only in the $i$ th position, $\mathbf{e}_{i}^{T} \mathbf{H}^{H} \mathbf{H} \mathbf{e}_{i}$ turns out to be equal to $4\left[\mathbf{H}^{H} \mathbf{H}\right]_{i, i}$. However, since $\mathbf{H}=\mathbf{D \Theta}$ and $\left|[\boldsymbol{\Theta}]_{i, i}\right|=1 / \sqrt{K},\left[\mathbf{H}^{H} \mathbf{H}\right]_{i, i}$ does not depends on $i$, and $\mathbf{e}_{i}^{T} \mathbf{H}^{H} \mathbf{H e}_{i}=4 \operatorname{tr}\left(\mathbf{D}^{H} \mathbf{D}\right) / K$. Hence, in order to find the most likely among the vectors $\left\{\hat{\mathbf{s}}+\mathbf{e}_{i}, i=1, \ldots, K\right\}$, it is sufficient to look for the maximum value assumed by $2 \operatorname{Re}\left(\mathbf{e}_{i}^{T} \mathbf{H}^{H}(\mathbf{y}-\mathbf{H} \hat{\mathbf{s}})\right)$, for $i=1, \ldots, K$. By defining the $K \times K$ diagonal matrix $\mathbf{E}=-2 \operatorname{diag}(\hat{\mathbf{s}})=\left[\mathbf{e}_{1}, \mathbf{e}_{2}, \ldots, \mathbf{e}_{K}\right]^{T}$, the LML detector has only to find the maximum value of the vector

$$
\mathbf{v}=2 \mathbf{E} \operatorname{Re}\left(\mathbf{H}^{H}(\mathbf{y}-\mathbf{H} \hat{\mathbf{s}})\right)
$$

and successively, if $\max (\mathbf{v})>4 \operatorname{tr}\left(\mathbf{D}^{H} \mathbf{D}\right) / K$, it has to flip the symbol of $\hat{\mathbf{s}}$ corresponding to the position of $\max (\mathbf{v})$. As a consequence of (16), the complexity of such an LML detector is comparable with that of decision-directed detectors. For other constellations, similar considerations hold true with minor modifications.

Before elaborating further on the computational complexity of the LML-MMSE detector, we first highlight that a unitary precoder also simplifies the MMSE detector computation, because the matrix to be inverted in (6) is diagonal. Moreover, we also point out that the LML detector can equivalently maximize the relative $L L F \Lambda(\hat{\mathbf{s}}+\mathbf{e})-\Lambda(\hat{\mathbf{s}})$, which is easier to be evaluated than $\Lambda(\hat{\mathbf{s}}+\mathbf{e})$, where, for a generic constellation, e represents an error vector containing at most $P$ non-zero values. Therefore, by plugging $\mathbf{e}$ in (15), and exploiting both $\mathbf{H}=\mathbf{D} \Theta$ and (14), it can be shown that the number of complex multiplications required for each received block $\mathbf{y}$ can be smartly reduced to

$$
N_{\text {mult }}=4 K \log _{2} K+8 K+\sum_{i=0}^{P}\left(i+i^{2}\right)(m-1)^{i}\left(\begin{array}{c}
K \\
i
\end{array}\right),
$$

which is equal to $N_{\text {mult }}=3 K^{2}+4 K \log _{2} K+7 K$ when $P=2$ and BPSK $(m=2)$ is used. Moreover, $K \log _{2} K+3 K-1$ additional complex multiplications are required at the beginning to compute $\mathbf{H}^{H} \mathbf{H}$, which has to be updated only when the channel changes. From (17), by focusing on the BPSK case and assuming $P \leq K / 2$, the computational complexity increases as $O\left(K^{P}\right)$. Thus, for $P=2$, the complexity of the LML-MMSE detector is below the average complexity of $\mathrm{SD}$, which is roughly $O\left(K^{3}\right)$ [15], and below the complexities of PDA and SDP, which are $O\left(K^{3}\right)$ [7], and $O\left(K^{3.5}\right)$ [6], respectively. This fact motivates the usefulness of the proposed algorithm with $P \leq 2$ for LP-OFDM systems. When $P>2$, the complexity of the LML-MMSE detector becomes comparable to those of near-ML algorithms, and hence a performance comparison with such techniques would be required (due to the lack of space, this comparison is not pursued here).

\section{Effect of a Pilot-Aided Channel Estimation Technique}

So far, we have implicitly assumed that the diagonal channel matrix $\mathbf{D}$, contained into $\mathbf{H}$, is known to the receiver. In practice, only an estimated version of $\mathbf{D}$ is available, and therefore the LML detector should be obtained by replacing the exact $\mathbf{H}$ with its estimate $\hat{\mathbf{H}}=\hat{\mathbf{D}} \boldsymbol{\Theta}$. In this subsection, our aim is to modify the MMSE detector of the first stage in order to take into account the channel estimation errors, and thereby improve the overall performance of the LML-MMSE detector.

We assume that $N_{\text {pil }}$ pilot subcarriers, equally spaced [18], are transmitted in the first OFDM block. Since we have assumed that the channel can have at most $L$ taps, we choose $N_{p i l}=L$. At the receiver, we assume ML channel estimation (MLE), which achieves the Cramér-Rao lower bound (CRLB) [19]. In this case, the covariance matrix of the frequencydomain channel estimation error $\underline{\boldsymbol{\varepsilon}}=\operatorname{diag}(\underline{\hat{\mathbf{D}}}-\underline{\mathbf{D}})$ can be expressed by $E\left\{\underline{\boldsymbol{\varepsilon}} \underline{\boldsymbol{\varepsilon}}^{H}\right\}=\sigma_{w}^{2} N N_{p i l}^{-1} \underline{\mathbf{F}} \underline{\mathbf{F}}^{\bar{H}}$, where $\underline{\mathbf{F}}$ is the $N \times N_{p i l}$ matrix obtained by selecting the first $N_{p i l}$ columns of $\mathbf{F}_{N}$. It turns out that if $K \in\left\{N_{p i l} / 2^{n}, n \in \mathbb{N}, n<\log _{2} N_{p i l}\right\}$, the covariance matrix of the channel estimation error in each precoded block is $E\left\{\boldsymbol{\varepsilon} \varepsilon^{H}\right\}=\sigma_{w}^{2} \mathbf{I}_{K}$. This implies that the channel estimation error can be interpreted as an additive white Gaussian error with the same power of the thermal AWGN represented by $\mathbf{w}$. In such a situation, we can define the modified MMSE (MMMSE) detector as in (6), where $\mathbf{H}$ and $\sigma_{w}^{2}$ are simply replaced by $\hat{\mathbf{H}}$ and $2 \sigma_{w}^{2}$, respectively. By similar considerations, we can also define the modified DFE (MDFE), and the modified PIC (MPIC), to be used when the channel is estimated by the aid of pilot tones.

\section{Simulation Results}

In this section, we present simulation results in order to assess the BER performance of the LML-MMSE detectors. We consider an OFDM system with cyclic prefix of length $L=16$, and $N=64$ subcarriers, spaced with one another by 312.5 $\mathrm{kHz}$. The chosen precoder is the one in (14). We use the channel model C of the IEEE 802.11a standard [16]. In this model, each tap suffers independent Rayleigh fading, with an exponentially decaying power delay profile and an rms delay spread equal to $150 \mathrm{~ns}$.

Figs. 1-2 show the BER performance of various detectors versus $E_{b} / N_{0}$, averaged over 400 channel realizations, when 
BPSK and a precoder size $K=8$ are used. Fig. 1 shows that at $\mathrm{BER}=10^{-3}$, the performance gain of the LML-MMSE detector with $P=1$ with respect to DFE and MMSE detector is roughly $1.4 \mathrm{~dB}$ and $2.8 \mathrm{~dB}$, respectively, while the loss with respect to the ML detector is approximately $1.2 \mathrm{~dB}$. Fig. 1 also indicates that 1 iteration of the LML search gives small performance improvement $\left(0.3 \mathrm{~dB}\right.$ when $\left.\mathrm{BER}=10^{-3}\right)$ with respect to the non-iterative LML-MMSE detector, at the expense of doubling the complexity. More iterations are not effective. Fig. 2 shows that the performance obtained by the LMLMMSE detector with $P>1$ is very close to those of ML detection. Although Fig. 1 suggests that the decision-directed detectors outperform the MMSE detector, Fig. 2 indicates that their LML counterparts behave differently. Indeed, when $P=1$, the LML-MMSE detector outperforms both the LML detector with DFE initialization (LML-DFE) and with PIC initialization (LML-PIC), with a gain of roughly $1 \mathrm{~dB}$ when $\mathrm{BER}=10^{-4}$. This fact is clearly explained by Fig. 3 , which plots versus $k$ the number of detected blocks with $k$ errors, when $2.88 \cdot 10^{6}$ blocks are transmitted at $E_{b} / N_{0}=10 \mathrm{~dB}$. Due to error propagation, the DFE produces a significant number of blocks with more than one symbol error, which are not recovered by a subsequent LML approach. On the contrary, most of the erroneous blocks of the MMSE detector contains only one error, and therefore in this case the LML approach with $P=1$ is quite effective.

Fig. 4 shows the BER performance, when QPSK with Gray coding and $K=16$ are used. In this case, the performance gains of the LML-MMSE detectors are smaller than in the previous case. This is due to the fact that for QPSK the cardinality of $S$ is higher than for BPSK. This means that in the QPSK case the LML search is performed in a smaller subset of the total set $S$, thus neglecting more possible solutions than for BPSK. However, also in this case the LML-MMSE detector with $P=1$ outperforms the DFE detector. Moreover, the RCLML-MMSE detectors with $D=\sqrt{2}$ achieve the same performance of their LML-MMSE counterparts, despite the smaller complexity (roughly half when $P=2$ ).

Fig. 5 compares the BER performance of MMSE, DFE, and PIC, with the ones of their modified counterparts, in the presence of channel estimation errors. BPSK, $N_{p i l}=16$, and $K=8$ are used. When BER $=10^{-4}$, the MMMSE provides 1.2 $\mathrm{dB}$ gain with respect to the MMSE. Fig. 6 depicts the BER of the LML-MMSE detector in the same simulation scenario of Fig. 5. It can be observed that the LML-MMSE approach seems to be effective also in the presence of channel estimation errors.

\section{CONCLUSIONS}

In this paper, we have considered the LML detection approach in LP-OFDM systems. We have shown that the output of the MMSE detector is a convenient initialization for the LML detector. The performance and complexity of the proposed LML-MMSE detector, which fall between the ones of the MMSE and ML detectors, can be nicely adjusted by controlling the neighborhood size. Simulation results have demonstrated that the LML-MMSE detector with minimum (nontrivial) neighborhood size outperforms the DFE approach, while exhibiting comparable complexity. Performance im- provements obtained by exploiting the soft information of the MMSE detector, as well as the thorough comparison with nearML techniques, will be the subject of future investigation.

\section{REFERENCES}

[1] R. van Nee and R. Prasad, OFDM for Wireless Multimedia Communications, Artech House, 2000.

[2] Z. Wang and G. B. Giannakis, "Complex-field coding for OFDM over fading wireless channels," IEEE Trans. Inform. Theory, vol. 49, pp. 707720, Mar. 2003.

[3] Z. Liu, Y. Xin, and G. B. Giannakis, "Linear constellation precoding for OFDM with maximum multipath diversity and coding gains," IEEE Trans. Commun., vol. 51, pp. 416-427, Mar. 2003.

[4] J. Boutros and E. Viterbo, "Signal space diversity: a power- and bandwidth-efficient diversity technique for the Rayleigh fading channel," IEEE Trans. Inform. Theory, vol. 44, pp. 1453-1467, July 1998.

[5] E. Viterbo and J. Boutros, "A universal lattice code decoder for fading channels," IEEE Trans. Inform. Theory, vol. 45, pp. 1639-1642, July 1999

[6] W.-K. Ma, T. N. Davidson, K. M. Wong, Z.-Q. Luo, and P.-C. Ching, "Quasi-maximum-likelihood multiuser detection using semi-definite relaxation with application to synchronous CDMA," IEEE Trans. Signal Processing, vol. 50, pp. 912-922, Apr. 2002.

[7] J. Luo, K. R. Pattipati, P. K. Willett, and F. Hasegawa, "Near-optimal multiuser detection in synchronous CDMA using probabilistic data association,” IEEE Commun. Lett., vol. 5, pp. 361-363, Sept. 2001.

[8] J. Hu and R. S. Blum, "A gradient guided search algorithm for multiuser detection," IEEE Commun. Lett., vol. 4, pp. 340-342, Nov. 2000.

[9] Y. Sun, "Local maximum likelihood multiuser detection for CDMA communications," in Proc. IEEE Int. Conf. Inform. Tech.: Coding and Computing, Las Vegas, NV, 2001, pp. 307-311.

[10] J. Luo, G. Levchuk, K. Pattipati, and P. Willett, "A class of coordinate descent methods for multiuser detection," in Proc. IEEE Int. Conf. Acoustic Speech Signal Processing, Istanbul, Turkey, 2000, vol. 5, pp. 2853-2856.

[11] Z. Wang and G. B. Giannakis, "Wireless multicarrier communications: where Fourier meets Shannon," IEEE Signal Processing Mag., vol. 17, pp. 29-48, May 2000.

[12] N. Al-Dhahir and A. H. Sayed, "The finite-length multi-input multioutput MMSE-DFE,” IEEE Trans. Signal Processing, vol. 48, pp. 29212936, Oct. 2000.

[13] A. Stamoulis, G. B. Giannakis, and A. Scaglione, "Block FIR decisionfeedback equalizers for filterbank precoded transmissions with blind channel estimation capabilities," IEEE Trans. Commun., vol. 49, pp. 6983, Jan. 2001.

[14] D. G. M. Cruickshank, "Suppression of multiple access interference in a DS-CDMA system using Wiener filtering and parallel cancellation," IEE Proc.-Commun., vol. 143, pp. 226-230, Aug. 1996.

[15] B. Hassibi and H. Vikalo, "Maximum-likelihood decoding and integer least-squares: the expected complexity," in Multiantenna Channels: Capacity, Coding and Signal Processing, G.J. Foschini and S. Verdú, Eds., American Mathematical Society, 2003.

[16] A. Doufexi et al., "A comparison of the HIPERLAN/2 and IEEE 802.11a wireless LAN standards," IEEE Commun. Mag., vol. 40, pp. 172-180, May 2002.

[17] Y.-P. Lin and S.-M. Phoong, "BER minimized OFDM systems with channel independent precoders," IEEE Trans. Signal Processing, vol. 51, pp. 2369-2380, Sept. 2003.

[18] R. Negi and J. Cioffi, "Pilot tone selection for channel estimation in a mobile OFDM system," IEEE Trans. Consum. Electron., vol. 44, pp. 1122-1128, Aug. 1998.

[19] M. Morelli and U. Mengali, "A comparison of pilot-aided channel estimation methods for OFDM systems," IEEE Trans. Signal Processing, vol. 49, pp. 3065-3073, Dec. 2001. 


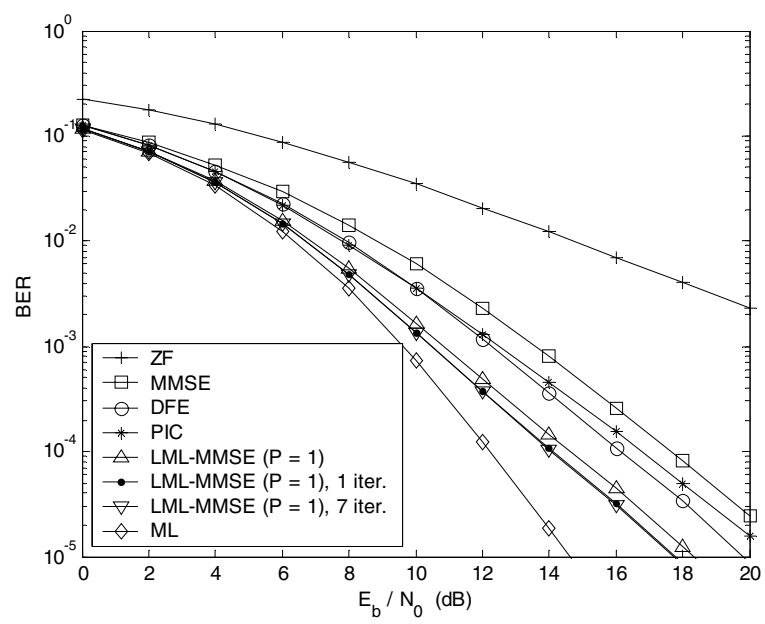

Figure 1. BER performance for BPSK.

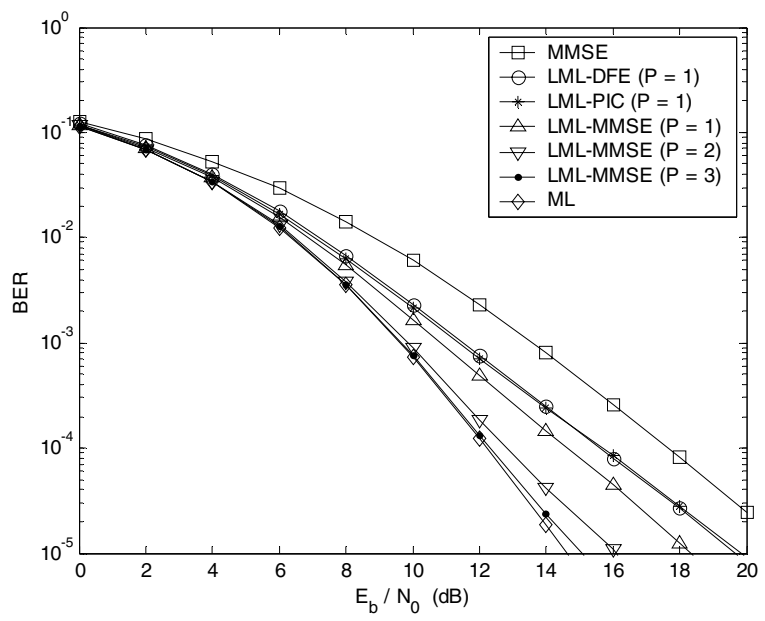

Figure 2. BER performance for BPSK.

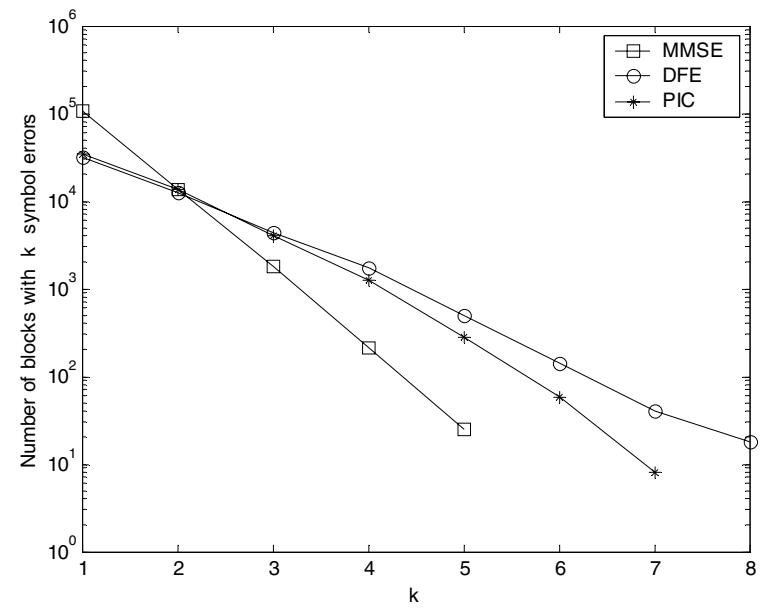

Figure 3. Distribution of the number of errors within the same data block.

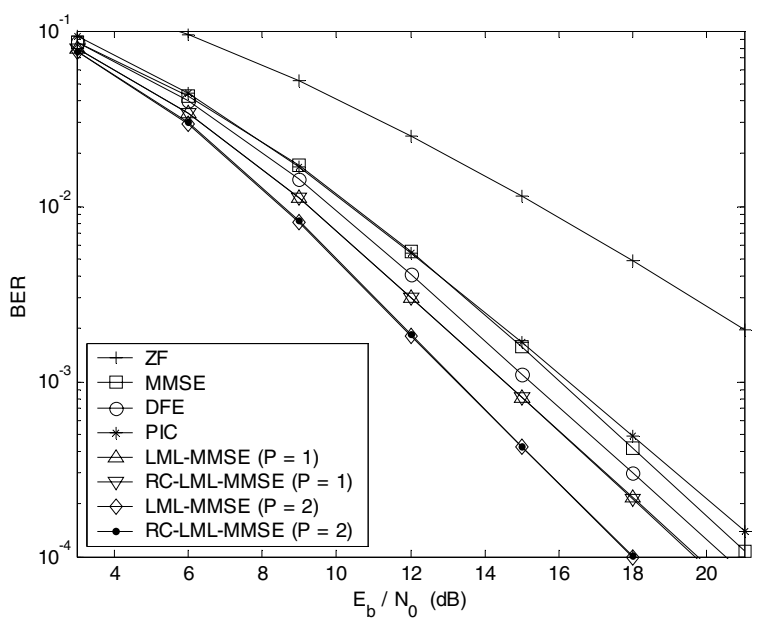

Figure 4. BER performance for QPSK.

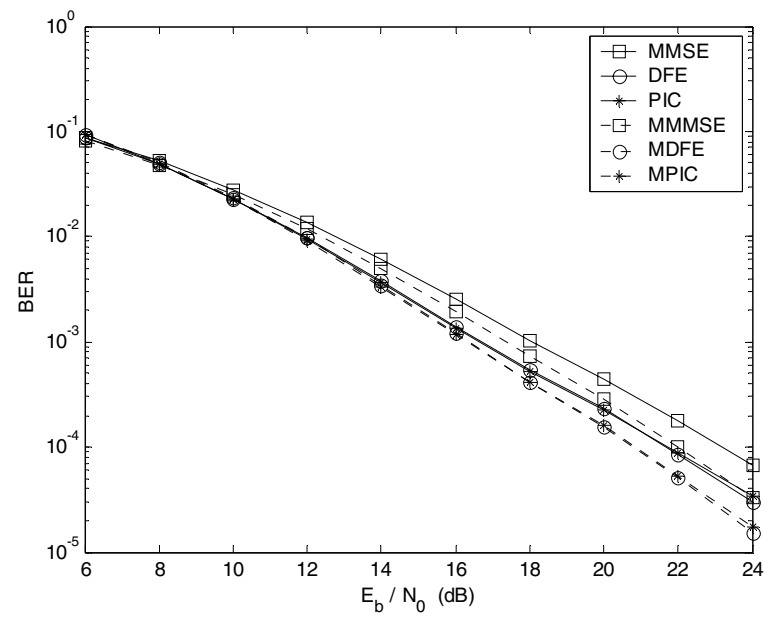

Figure 5. BER performance with channel estimation errors.

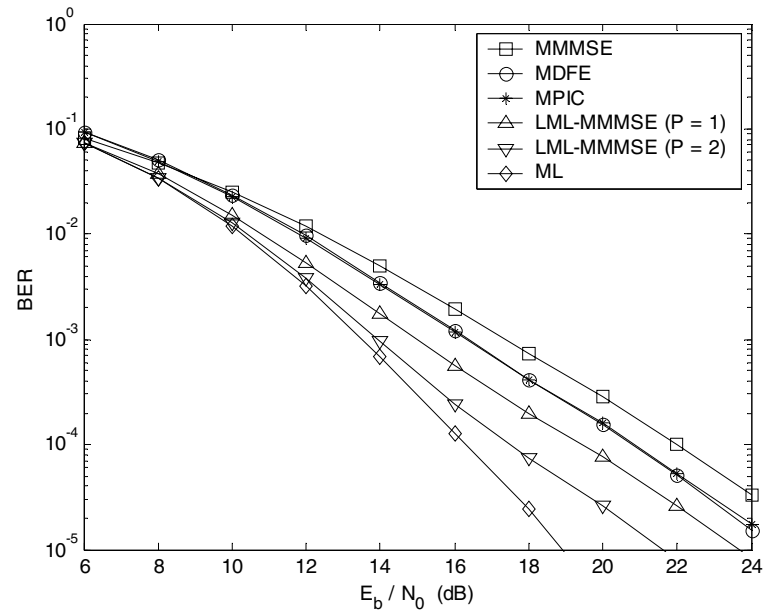

Figure 6. BER performance with channel estimation errors. 